\title{
Ultrahigh power-bandwidth-product performance of low-temperature-grown-GaAs based metal-semiconductor-metal traveling-wave photodetectors
}

\author{
Kian-Giap Gan \\ Department of Electrical and Computer Engineering, University of California, Santa Barbara, \\ California 93106-5050 \\ Jin-Wei Shi and Yen-Hung Chen \\ Graduate Institute of Electro-Optical Engineering, National Taiwan University, Taipei 10617, Taiwan \\ Chi-Kuang Sun ${ }^{\text {a) }}$ \\ Graduate Institute of Electro-Optical Engineering and Department of Electrical Engineering, National \\ Taiwan University, Taipei 10617, Taiwan \\ Yi-Jen Chiu and John E. Bowers \\ Department of Electrical and Computer Engineering, University of California, Santa Barbara, \\ California $93106-5050$
}

(Received 12 December 2001; accepted for publication 1 April 2002)

\begin{abstract}
High-output-power and high-bandwidth performances are usually two tradeoff parameters in the design of high-speed photodetectors. In this letter, we report high peak-output-voltage $(\sim 20 \mathrm{~V})$ and peak-output-current ( $\sim 400 \mathrm{~mA}, 50 \Omega$ load) together with ultrahigh-speed performances $(1.5 \mathrm{ps}, 220$ $\mathrm{GHz}$ ), observed in low-temperature-grown-GaAs (LTG-GaAs) based metal-semiconductor-metal (MSM) traveling-wave photodetectors (TWPDs) at a wavelength of $800 \mathrm{~nm}$. Ultrahigh-peak-output-power and ultrahigh-electrical-bandwidth performances were achieved due to the superior MSM microwave guiding structure and a short carrier trapping time in the LTG-GaAs layer, which reduced the space-charge screening effect and increased the photoabsorption volume without sacrificing electrical bandwidth significantly. We also observed different bias-dependent nonlinear behaviors in MSM TWPDs under high and low illuminated optical power excitations, which are possibly dominated by the space-charge screening and the lifetime increasing effects, respectively. (c) 2002 American Institute of Physics.
\end{abstract}

[DOI: $10.1063 / 1.1482139]$

Ultrahigh speed photodetectors (PDs) attract much attention due to their applications in fiber communication and optical measurement systems. ${ }^{1}$ Recently, the development of optical amplifier has created a new demand of high inputoptical power durability in these high-speed PDs. Some receiver circuits use fiber amplifiers as preamplifiers, which relieve or even eliminate the necessity of electrical amplifiers. ${ }^{1}$ By utilizing photomixing techniques, microwave photonic systems are expected to generate tunable and highpower microwaves or submillimeter waves under intense optical excitation. ${ }^{2}$ However, ultrahigh bandwidth PDs are known to suffer bandwidth degradation problems under intense optical illumination. ${ }^{3,4}$ Maximum output current/power and electrical bandwidth performances are usually two tradeoff parameters in the design of high-speed PDs. ${ }^{1}$ By properly scaling down the size and photoabsorption volumes of PDs, ultrahigh speed performances can be achieved due to the reduction of parasitic capacitance and resistance in PDs. ${ }^{5}$ However, the small photoabsorption volume $\left(\sim 1 \mu \mathrm{m}^{3}\right)$ would cause high densities of photogenerated free carriers inducing strong space-charge fields that screen the external applied bias field. The device electrical bandwidth would thus degrade seriously due to the reduction in drift velocities of photogenerated carriers. ${ }^{3,4}$ On the other hand, by reducing

\footnotetext{
a) Author to whom correspondence should be addressed; electronic mail: sun@cc.ee.ntu.edu.tw
}

the optical modal absorption constant and increasing the photoabsorption volume, the output power can be increased significantly at the expense of the electrical bandwidth limited by $\mathrm{R}-\mathrm{C}$ time constant and high microwave loss in the large-area and long-absorption-length devices. ${ }^{6,7}$ There are two major ways to increase the output-saturationcurrent(power)-electrical-bandwidth-product performances. One is to distribute the photocurrents along edge-coupled PDs, such as the velocity match distributed photodetectors (VMDPs) ${ }^{8}$ the other is to increase the carrier velocity using unitraveling carrier PDs (UTC-PDs). ${ }^{1}$ In this letter, we demonstrate the high power performance in an ultrahigh speed low-temperature-grown GaAs (LTG-GaAs) based metalsemiconductor-metal (MSM) traveling-wave photodetector (TWPD). ${ }^{9}$ By utilizing the MSM microwave guiding structure, a large photoabsorption volume can be achieved without serious electrical bandwidth degradation. ${ }^{7}$ The spacecharge screening effect in most high power PDs could also be reduced with the LTG-GaAs photoabsorption layer due to its short carrier trapping time and due to the fact that most of the collected photogenerated carriers are from the region near the metal contacts, where least space-charge screening effects could occur. ${ }^{1,4}$ Performance with record-high peakoutput-voltage (current) bandwidth product $[20 \mathrm{~V}(400 \mathrm{~mA}$ peak output current at $50 \Omega$ load), $220 \mathrm{GHz}, 4.4 \mathrm{THz}-\mathrm{V}$ (88 GHz-A)] is thus observed in the demonstrated MSM TWPD. Distinct bias-dependent nonlinear behavior has also been ob- 


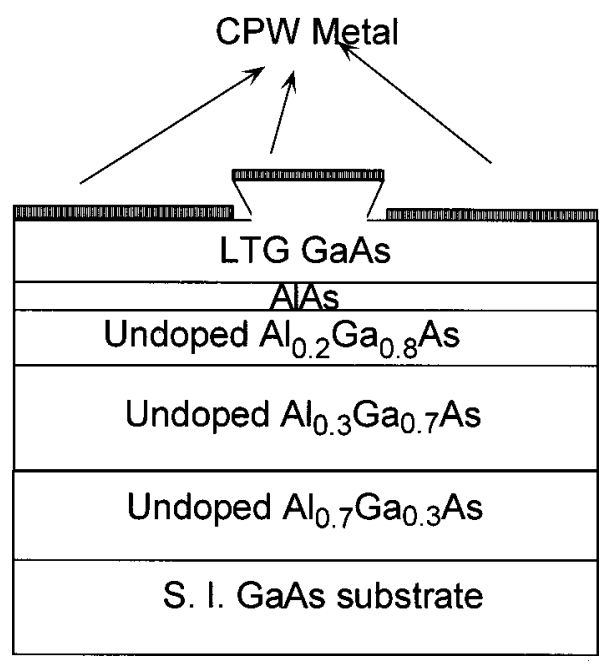

FIG. 1. Cross sectional diagram of MSM-TWPD.

served under different excitation power levels, which are dominated by the lifetime increasing effect ${ }^{10}$ or a combination of the defect saturation and space-charge screening effects $^{11,12}$ under low and high optical radiation powers, respectively.

The structure of the measured MSM-TWPDs is similar to the structure shown in Ref. 9 except for the AlGaAs optical cladding layers, which were modified to reduce substrate mode for better optical guiding. The cross sectional diagram is shown in Fig. 1. The thickness of each layer from surface to the bottom semi-insulating GaAs substrate is $500 \mathrm{~nm}$ of LTG-GaAs, $100 \AA$ of AlAs, $400 \mathrm{~nm}$ of $\mathrm{Al}_{0.2} \mathrm{Ga}_{0.8} \mathrm{As}, 1 \mu \mathrm{m}$ of $\mathrm{Al}_{0.3} \mathrm{Ga}_{0.7} \mathrm{As}$, and $3 \mu \mathrm{m}$ of $\mathrm{Al}_{0.7} \mathrm{Ga}_{0.3}$ As. All samples were annealed in molecular beam epitaxy chamber at $600{ }^{\circ} \mathrm{C}$ with detailed fabrication processes given in Ref. 9. By measuring devices with different absorption lengths, the waveguide length for complete absorption was determined to be $20 \mu \mathrm{m}$. Compared with the performance of traditional $p-i-n$ TWPDs with a "slow-wave" microwave mode, ${ }^{13}$ MSM TWPD structure ensures higher velocity-mismatch bandwidth ${ }^{14}$ not only due to lower microwave propagation loss but also due to higher propagation velocity with the "quasi-TEM" mode. ${ }^{713}$ Because of superior microwave guiding structure, MSM TWPDs can increase photoabsorption volume and output power with less electrical bandwidth degradation than $p-i-n$ TWPDs.

The detector's impulse current response was measured using an electro-optical (EO) sampling technique ${ }^{15}$ based on a femtosecond Ti:sapphire laser at $800 \mathrm{~nm}$ with $100 \mathrm{MHz}$ repetition rate. Figure 2 shows the full width at half maximum (FWHM) of the EO measured electrical impulse response in a $10-\mu \mathrm{m}$-long MSM TWPD as a function of bias voltage for different optical pumping energies. The waveguide width of the measured device is $2 \mu \mathrm{m}$. Although the short device length $(10 \mu \mathrm{m})$ cannot absorb the input optical energy completely, it ensures that internal carrier dynamic instead of microwave property limits our measured device responses. ${ }^{7}$ We can clearly see that in trace $\mathrm{F}$, which corresponds to the lowest optical pumping energy $(0.49 \mathrm{pJ} / \mathrm{pulse})$, device response time increases when the bias voltage is over $2 \mathrm{~V}$. However, in trace A of the highest optical pumping energy (79 $\mathrm{pJ} /$ pulse), device response time decreases with

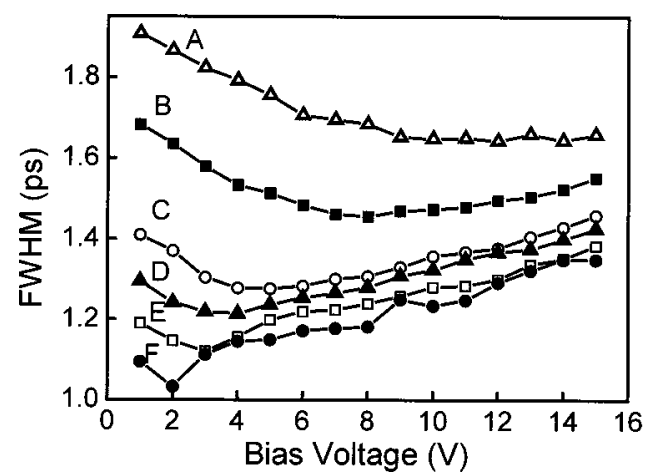

FIG. 2. FWHM of EO measured transient responses of a 10- $\mu \mathrm{m}$-long MSM TWPD under different optical excitation energies and bias voltage $(1-15 \mathrm{~V})$ levels. Traces A-F represent different optical excitation energies, which are $79,45,17,9.0,4.1$, and $0.49 \mathrm{pJ} /$ pulse, respectively.

the bias voltage. In other intermediate optical pumping energies, as shown in traces of $\mathrm{B}-\mathrm{E}$, an optimal bias point for the fastest device response exists. When the optical excitation energy increases, the optimal operation bias will also increase. We attribute this nonlinear behavior to the combination of different physical processes including carrier lifetime increasing, ${ }^{10}$ defect saturation, and space-charge screening effects. ${ }^{11,12}$ The device effective response time $t_{\text {eff }}$ due to carrier transport can be expressed as

$$
\frac{1}{t_{\text {eff }}}=\frac{1}{t_{\text {trapping }}}+\frac{1}{t_{\text {drift }}}
$$

where $t_{\text {trapping }}$ represents the carrier trapping time in LTGGaAs layer, which has a time scale in the subpicosecond range. ${ }^{16} t_{\text {drift }}$ is the carrier drift time in the LTG-GaAs photoabsorption layer, which is on the order of 8 ps by assuming a carrier saturation velocity and a drift distance of 5 $\times 10^{4} \mathrm{~m} / \mathrm{s}$ and $400 \mathrm{~nm}$, respectively. ${ }^{1,10} t_{\text {trapping }}$ and $t_{\text {drift }}$ are both functions of the applied electric field and the photogenerated carrier density. In the low optical excitation regime, $t_{\text {trapping }}$ increases with the bias voltage due to electron heating and Coulomb-barrier lowering. ${ }^{10,17}$ When the photoexcited carrier density increases, carrier trapping time will increase significantly because defect saturation reduces the carrier capture probability. Regarding $t_{\text {drift }}$, carrier drift time can be reduced significantly with increased applied electric field until the carriers arrive at their saturation velocity. However, under high illumination, the drifted high-density carriers will induce a significant space-charge field to screen out the external applied field and slow down the carrier velocity, which will increase $t_{\text {drift }}$. In trace $\mathrm{F}$, the density of photogenerated carriers $\left(\sim 7 \times 10^{16} \mathrm{~cm}^{-3}\right)$ is much smaller than the defect density in LTG-GaAs layer $\left(\sim 8 \times 10^{17} \mathrm{~cm}^{-3}\right)^{18}$ and $t_{\text {trapping }}$, which increases with bias voltage as previously discussed, instead $t_{\text {drift }}$ dominates the measured response time. When optical pumping power increases, $t_{\text {trapping }}$ also increases to the order of picoseconds ${ }^{11}$ that is close to the order of $t_{\text {drift }}$, and the variation of $t_{\text {drift }}$ will start to affect the measured FWHM. In trace C, for example, the measured FWHM decreases with the bias voltage below $4 \mathrm{~V}$ due to the reduction in $t_{\text {drift }}$. However with further increased bias voltage, the FWHM broadens again due to lifetime increasing effect. As shown in traces $\mathrm{B}, \mathrm{C}$, and $\mathrm{D}$, these optimal bias points for narrowest FWHM shift toward higher voltages for higher 


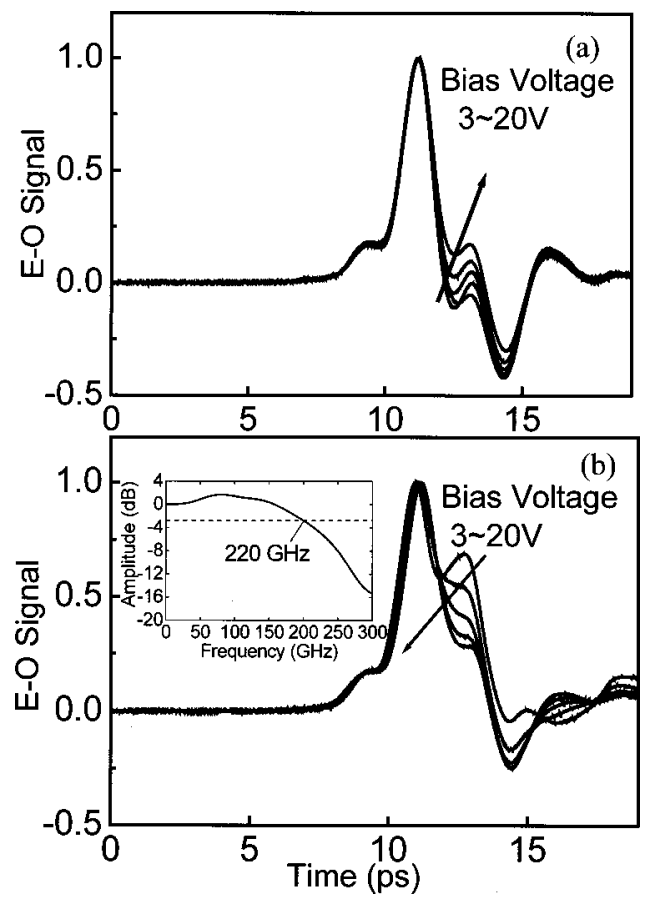

FIG. 3. (a) EO measured bias dependent (3-20 V) transient responses of a $23-\mu \mathrm{m}$-long MSM TWPD with a low optical excitation energy $(8.8 \mathrm{pJ} /$ pulse) and (b) with a high optical excitation energy (76 pJ/pulse); (b) shows the corresponding frequency domain response of the 20-V-bias trace by using a fast-Fourier transform technique, which shows a 220-GHz-electrical bandwidth with $20 \mathrm{~V}$ peak-output-voltage and $400 \mathrm{~mA}$ peak-output-current with a $50 \Omega$ load.

optical pumping energies. The shift of optimal bias voltage is due to that fact that a higher electric field is needed to overcome the carrier-induced space-charge field, which increases with the generated carrier densities.

In order to further increase the output power from the MSM TWPD, longer absorption length is required. Figure 3(a) shows the measured bias dependent impulse responses of a 23- $\mu \mathrm{m}$-long MSM TWPD under low optical energy excitation $(8.8 \mathrm{pJ} / \mathrm{pulse})$ with normalized peak intensity. This long-absorption-length MSM TWPD has a much larger photoabsorption volume $\left(\sim 23 \mu \mathrm{m}^{3}\right)$ than the volume of previous ultrahigh speed PDs $\left(\sim 1 \mu \mathrm{m}^{3}\right) .{ }^{8}$ The FWHM of the measured responses is almost the same as the $10-\mu \mathrm{m}$-long device, which implies that a large photoabsorption volume can be achieved without significantly sacrificing electrical bandwidth. The quantum efficiency $(2.7 \%)$ of this longer device is about 1.7 times larger than the $10-\mu \mathrm{m}$-long device $(1.5 \%)$ at a $15 \mathrm{~V}$ bias voltage. Regarding its high power performances, the measured bias dependent impulse responses are also shown in Fig. 3(b) with normalized peak intensity. The optical excitation energy is $76 \mathrm{pJ} / \mathrm{pulse}$. By increasing the bias voltage, nonlinear saturation behavior can be reduced because of increased external bias field as discussed before. The collected photogenerated charge and its corresponding peak output photocurrent $I_{p}$ at $20 \mathrm{~V}$ bias voltage are about $1600 \mathrm{fC}$ and $\sim 400 \mathrm{~mA}$, respectively. We calculate the peak output photocurrent by the area of the normalized E-O signal trace and the collected photogenerated charge per pulse. By multiplying the peak-output-photocurrent with the characteristic impedance of integrated CPW line $(50 \Omega)$, we can also get the peak-output-voltage $V_{p}$. The calculated $V_{p}$ for the $20 \mathrm{~V}$ bias trace (under $76 \mathrm{pJ} /$ pulse excitation) is also around $20 \mathrm{~V}$, which is obviously limited by the external applied bias. Its corresponding frequency response with 220 $\mathrm{GHz}$ electrical $3 \mathrm{~dB}$ bandwidth is shown in the inset of Fig. 3(b), which was obtained by the fast Fourier transform technique. The $V_{p}(20 \mathrm{~V}) / I_{p}(400 \mathrm{~mA})$-electrical bandwidth (220 $\mathrm{GHz}$ ) products of $4.4 \mathrm{THz}-\mathrm{V} / 88 \mathrm{GHz}-\mathrm{A}$ are both the highest among all the reported ultrahigh speed PDs, including LTGGaAs $p-i-n$ TWPDs (6 ps, $1400 \mathrm{fC}){ }^{12}$ InGaAs vertical $p-i-n$ PD (7.2 ps, $68 \mathrm{fC}),{ }^{19}$ GaAs $p-i-n$ TWPD (5.5 ps, $59 \mathrm{fC}),{ }^{20} \mathrm{UTC}$ PD $\left(3.1 \mathrm{ps}, 115 \mathrm{GHz}, V_{p}: 1.92 \mathrm{~V}, 76 \mathrm{~mA}\right.$, $25 \Omega$ load $),{ }^{1}$ and VMPD $\left(50 \mathrm{GHz}, V_{p}: 2.5 \mathrm{~V}\right){ }^{8}$

In conclusion, a record performance of peak-outputvoltage (current)-electrical bandwidth product $(4.4 \mathrm{THz}-\mathrm{V}$ (88 GHz-A): $220 \mathrm{GHz}, 20 \mathrm{~V}, 400 \mathrm{~mA}$ at $50 \Omega$ load) has been demonstrated using a MSM TWPD structure under $800 \mathrm{~nm}$ wavelength excitation. Different nonlinear behaviors of LTG-GaAs under low and high optical power illuminations have been observed, which are attributed to the lifetime increasing effect and the combination of defect saturation with the space-charge screening effect. The ultrahigh speed and high power performances of our demonstrated devices can find applications in microwave photonic systems, ${ }^{2}$ high power photomixers, ${ }^{21}$ and photoreceiver circuits without electrical amplifiers. ${ }^{1}$

This work is sponsored by National Science Council of Taiwan under Grant No. NSC89-2215-E-002-064 and National Science Foundation of USA under Award No. INT9813411.

${ }^{1}$ K. Kato, IEEE Trans. Microwave Theory Tech. 47, 1265 (1999)

${ }^{2}$ S. Fukushima, C. F. C. Silva, Y. Muramoto, and A. J. Seeds, IEEE Photonics Technol. Lett. 13, 720 (2001).

${ }^{3}$ K. J. Williams, R. D. Esman, and M. Dagenais, J. Lightwave Technol. 14, 84 (1996).

${ }^{4}$ Y.-L. Huang and C.-K. Sun, J. Lightwave Technol. 18, 203 (2000).

${ }^{5}$ H. Ito, T. Furuta, S. Kodama, N. Watanabe, and T. Ishibashi, Electron. Lett. 36, 1809 (2000).

${ }^{6}$ V. M. Hietala, G. A. Vawter, T. M. Brenan, and B. E. Hammons, IEEE Trans. Microwave Theory Tech. 43, 2291 (1995).

${ }^{7}$ J.-W. Shi and C.-K. Sun, J. Lightwave Technol. 18, 2176 (2000).

${ }^{8}$ L. Y. Lin, M. C. Wu, T. Itoh, T. A. Vang, R. E. Muller, D. L. Sivco, and A. Y. Cho, IEEE Trans. Microwave Theory Tech. 45, 1320 (1997).

${ }^{9}$ J.-W. Shi, K. G. Gan, Y. J. Chiu, Y.-H. Chen, C.-K. Sun, Y. J. Yang, and J. E. Bowers, IEEE Photonics Technol. Lett. 16, 623 (2001).

${ }^{10}$ N. Zamdmer, Q. Hu, K. A. McIntosh, and S. Verghese, Appl. Phys. Lett. 75, 2313 (1999).

${ }^{11}$ A. J. Lochtefeld, M. R. Melloch, J. C. P. Chang, and E. S. Harmon, Appl. Phys. Lett. 69, 1465 (1996).

${ }^{12}$ Y. J. Chiu, S. B. Fleischer, J. E. Bowers, A. C. Gossard, and U. K. Mishra, Conference on Lasers and Electro-Optics, OSA Technical Digest (1998), p. 501 .

${ }^{13}$ H. Hasegawa, M. Furukawa, and H. Yanai, IEEE Trans. Microwave Theory Tech. 19, 869 (1971).

${ }^{14}$ K. S. Giboney, M. J. W. Rodwell, and J. E. Bowers, IEEE Photonics Technol. Lett. 4, 1363 (1992).

${ }^{15}$ K. J. Weingarten, M. J. W. Rodwell, and D. M. Bloom, IEEE J. Quantum Electron. 24, 198 (1988).

${ }^{16}$ S. Gupta, J. F. Whitaker, and G. A. Mourou, IEEE J. Quantum Electron. 28, 2464 (1992).

${ }^{17}$ T. H. Ning, J. Appl. Phys. 47, 3203 (1976).

${ }^{18}$ J. P. Ibbetson and U. K. Mishra, Appl. Phys. Lett. 68, 3781 (1996).

${ }^{19}$ C.-K. Sun, I.-H. Tan, and J. E. Bowers, IEEE Photonics Technol. Lett. 10, 135 (1998).

${ }^{20}$ K. S. Giboney, M. J. Rodwell, and J. E. Bowers, IEEE J. Sel. Top. Quantum Electron. 2, 622 (1996).

${ }^{21}$ S. Verghese, K. A. McIntosh, and E. R. Brown, IEEE Trans. Microwave Theory Tech. 45, 1301 (1997). 
Applied Physics Letters is copyrighted by the American Institute of Physics (AIP). Redistribution of journal material is subject to the AIP online journal license and/or AIP copyright. For more information, see http:/ojps.aip.org/aplo/aplcr.jsp

Copyright of Applied Physics Letters is the property of American Institute of Physics and its content may not be copied or emailed to multiple sites or posted to a listserv without the copyright holder's express written permission. However, users may print, download, or email articles for individual use. 